\title{
Research of ID Card Recognition Algorithm Based on Neural Network Pattern Recognition
}

\author{
Wang Naiguo,Zhu Xiangwei* \\ Suzhou Industrial Park Branch \\ Jiangsu Union Technical Institute \\ Suzhou, China \\ zxwook@163.com*corresponding author
}

\author{
Zhang Jian \\ School of computer Science \& Technology \\ Soochow University \\ Suzhou, China \\ zhangjian2012@suda.edu.cn
}

\begin{abstract}
How to quickly and correctly identify the second generation ID card code has important practical significance in real life. The algorithms have been presented about the recognition and verification code of ID cards. In order to let computer own the knowledge of code of character image, firstly, transform the image of ID cards to binary image, then segment image character of ID card code and display image character using image processing technique, secondly manual input corresponding correct code of character image, thirdly character image is classification stored in the corresponding folder according to the corresponding correct code. These character images are training samples, which are trained using the matlab toolbox of the neural network pattern to generate training model. Using the generated the training model to identify code of the ID cards. The validation algorithm is based on the generated the training model to identify code of ID cards. Compare the consistent of code of ID cards to identify the code, if recognition accuracy rate is not high, the training model need to be trained again, until the recognition accuracy rate is more than $95 \%$. Neural network pattern recognition and verification algorithm can take advantage of the training model to perfectly realize identification and validation the code of the second-generation ID card, the algorithms have application value in the real work.
\end{abstract}

Keywords- neural network; pattern recognition; image processing; ID card; segmentation

\section{INTRODUCTION}

ID is a unique identifier resident status, identity cards are widely used in identity testing department needs the service industry, transportation systems and public security system, it has become essential for the life of the certificates, which plays an important role in the personnel management. At present the registration ID card usually adopts manual fill or manual entry, the work efficiency is low, and the number of erroneous input possibility is big, and the use of computers to automatically identify entry ID can save a lot of manpower and resources, which has broad application prospects.

The algorithm has been proposed using the image processing technology and neural network pattern recognition technology in this paper. It realizes the automatic recognition of the identity card code. The result of experiment illustrates recognition accuracy rate more than $97 \%$ if the image ID code is clear. The algorithm fully meets the actual demand of automatic recognition ID code.

\section{BP NEURAL NETWORK}

In machine learning and cognitive science, artificial neural networks (ANNs) are a family of statistical learning algorithms inspired by biological neural networks and are used to estimate or approximate functions that can depend on a large number of inputs and are generally unknown. Artificial neural networks are generally presented as systems of interconnected "neurons" which can compute values from inputs, and are capable of machine learning as well as pattern recognition thanks to their adaptive nature.

The BPNN (Back Propagation Neural Network) is a special feed-forward network including an input layer, a hidden layer and an output layer as shown in Fig .1.

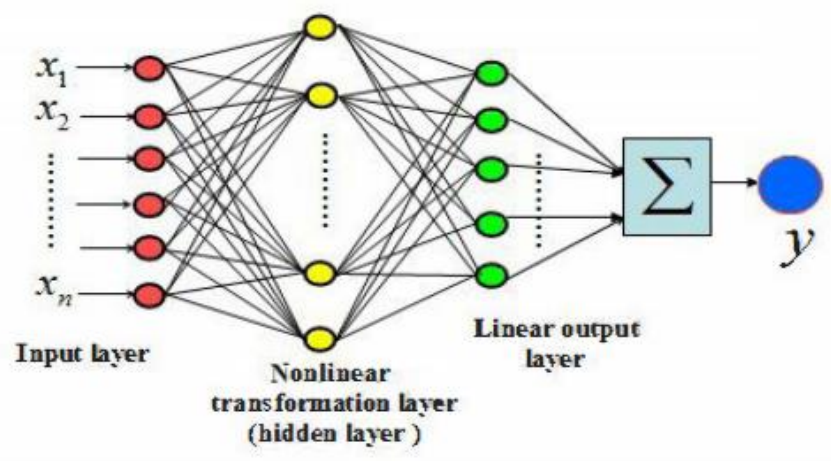

Figure 1. Back Propagation Neural Network architecture

The input layer passes the input signal to the hidden space. There is only one hidden node and its basis function is Gaussian kernel function. It realizes the non-linear conversion from input space to hidden space. The mapping from hidden space to output space is linear and the output of the network is linear weighed sum of the output of hidden unit.

Fig .1 presents part of a typical multilayer network workflow. For more information and other steps, see Multilayer Neural Networks and Back Propagation Training.

When the network weights and biases are initialized, the network is ready for training. The multilayer feed forward network can be trained for function approximation (nonlinear regression) or pattern recognition. The training process requires a set of examples of proper network behavior - network inputs and target outputs.

The process of training a neural network involves tuning the values of the weights and biases of the network to optimize network performance, as defined by the 
network performance function net(performFc). The default performance function for feed forward networks is mean square error mse(the average squared error) between the network outputs a and the target outputs t. It is defined as following equation (1):

$$
M S E=\frac{1}{N} \sum_{i=1}^{N}\left(e_{i}\right)^{2}=\frac{1}{N} \sum_{i=1}^{N}\left(t_{i}-a_{i}\right)^{2}
$$

There are two different ways in which training can be implemented: incremental mode and batch mode. In incremental mode, the gradient is computed and the weights are updated after each input is applied to the network. In batch mode, all the inputs in the training set are applied to the network before the weights are updated. Incremental training with the adapt command is discussed in Incremental Training with adapt. For most problems, when using the Neural Network Toolbox ${ }^{\mathrm{TM}}$ software, batch training is significantly faster and produces smaller errors than incremental training.

For training multilayer feed forward networks, any standard numerical optimization algorithm can be used to optimize the performance function, but there are a few key ones that have shown excellent performance for neural network training. These optimization methods use either the gradient of the network performance with respect to the network weights.

The gradient is calculated using a technique called the back propagation algorithm, which involves performing computations backward through the network. The back propagation computation is derived using the chain rule of calculus.

\section{TRAINING ALGORITHM AND RESULT}

\section{A. Sample Collection of Identity Card}

In order to generate the training mode, identity card samples should be collected. One of the ID samples is shown in Fig .2.

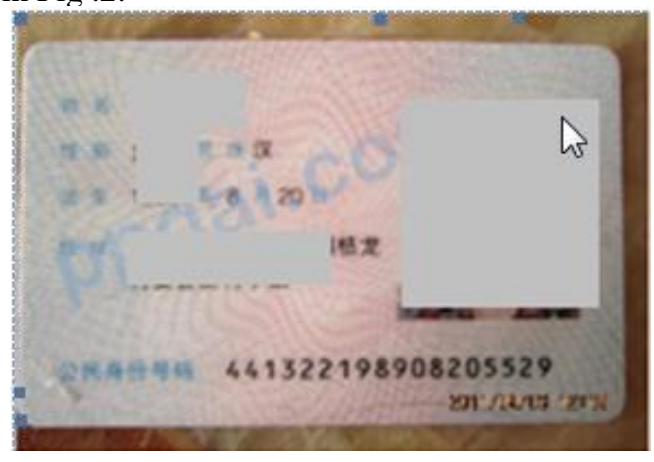

Figure 2. one of the ID card samples

\section{B. Genreate Segmentation Character of ID Code}

TABLE I illustrates the steps and results of segmentation character of ID code.

TABLE I. STEPS SEGMENTATION CHARACTER OF ID CODE

\section{Training Model According to the Character Images}

According to character images are classification stored in the corresponding folder perform to train model.

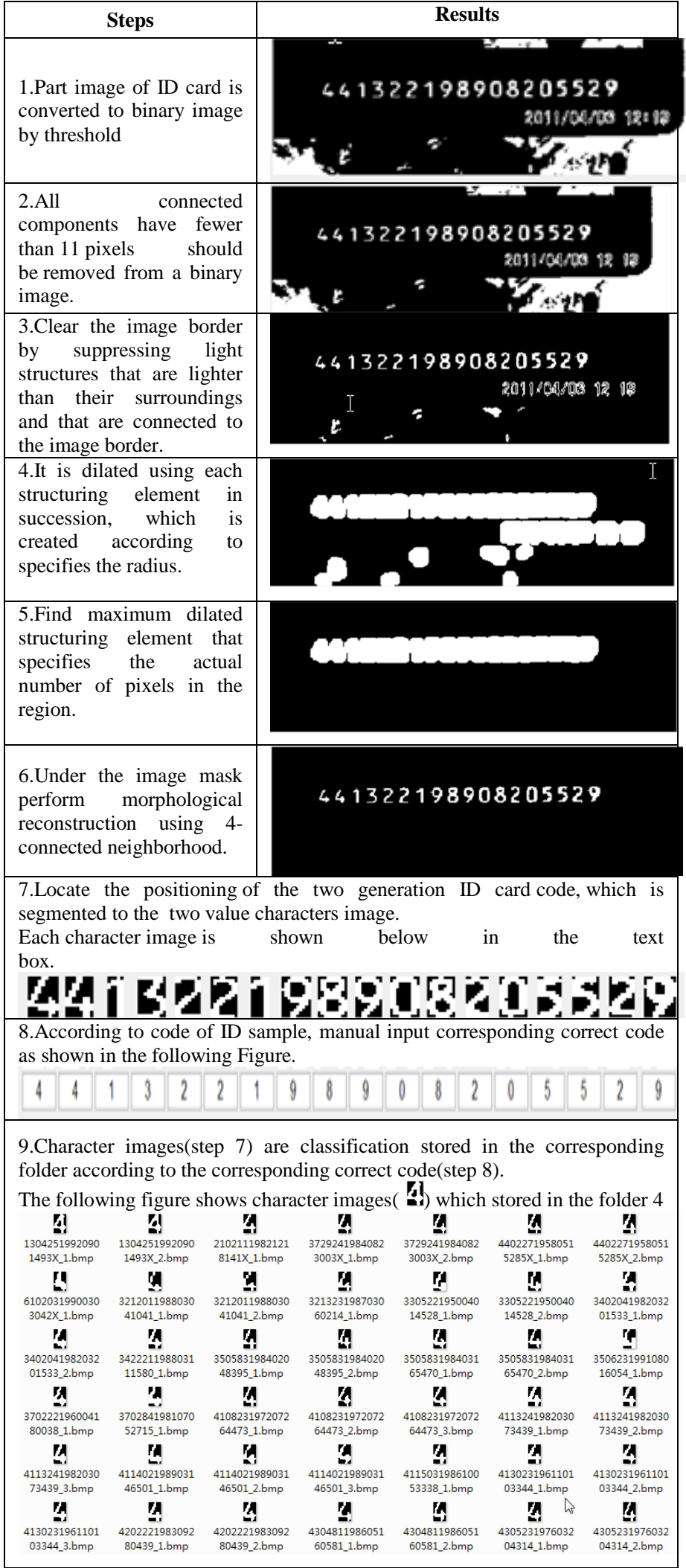

1) Run the toolbox of the neural network pattern recognition, and then fill neural network parameters of inputs and outputs as shown Fig.3. 


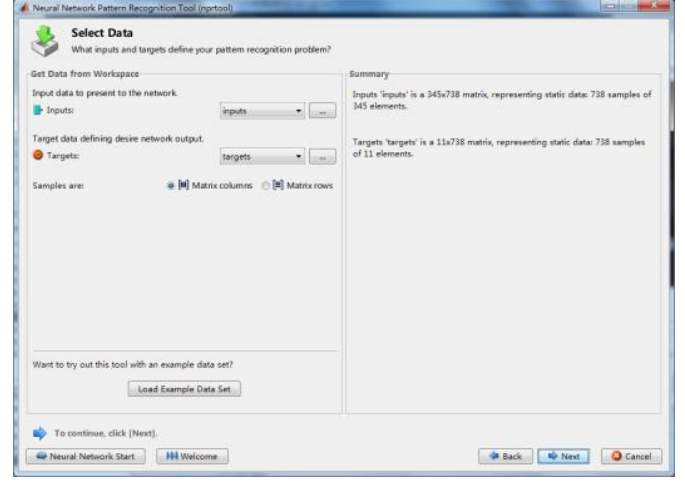

Figure 3. neural network parameters

2) Validation and test data sets are each set to $15 \%$ of the original data. With these settings, the input vectors and target vectors will be randomly divided into three sets as shown Fig .4.

a) $70 \%$ are used for training;

b) $15 \%$ are used to validate that the network is generalizing and to stop training before over fitting;

c) The last $15 \%$ are used as a completely independent test of network generalization.

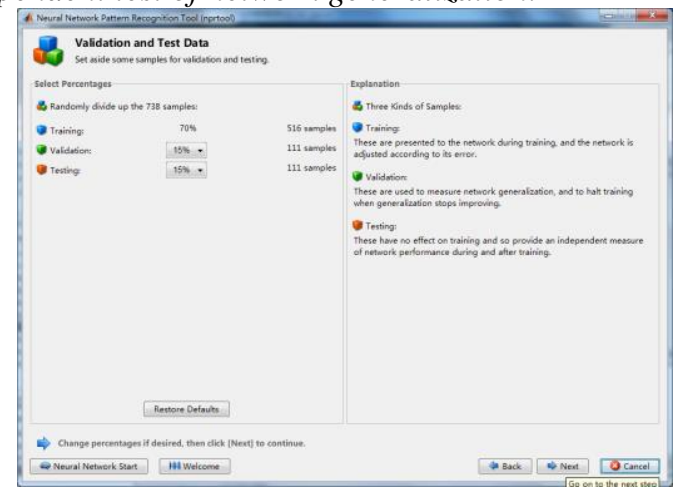

Figure 4. Training, Validation and Test data sets

3) Hidden number of neurons have a great impact on the generation of the training model, so select the appropriate value in the course of the experiment is very important. Through repeated experiments, define number of hidden neurons to 27 as shown Fig .5.

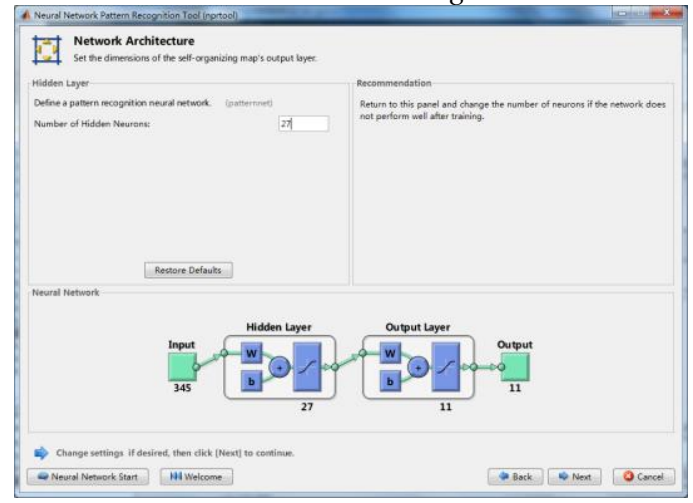

Figure 5. define number of hidden neurons

4) Train the network to classify the inputs according to the targets using scaled conjugate gradient back propagation, and then generate the training model as shown Fig .6.

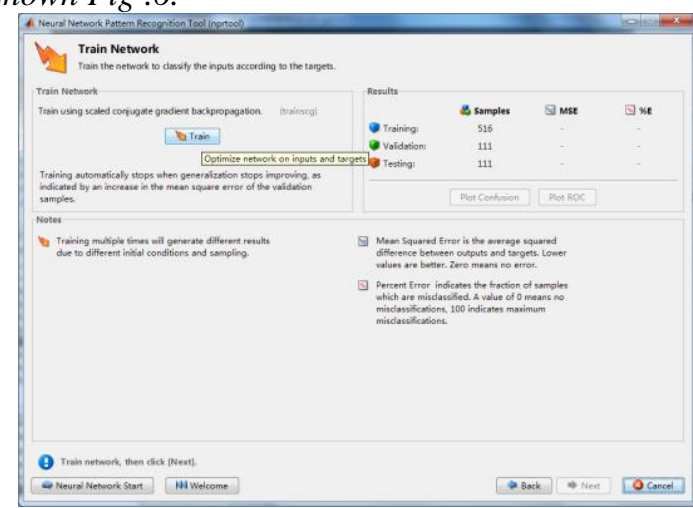

Figure 6. train the network to generate training model

The algorithm of scaled conjugate gradient back propagation is the simplest optimization algorithm. It updates the network weights and biases in the direction in which the performance function decreases most rapidly, the negative of the gradient. One iteration of this algorithm can be written as following equation (2):

$$
\mathbf{x}_{k+1}=\mathbf{x}_{k}-\alpha_{k} \mathbf{g}_{k}
$$

Where $\boldsymbol{x}_{k}$ is a vector of current weights and biases, $\boldsymbol{g}_{k}$ is the current gradient, and $\alpha_{k}$ is the learning rate. This equation is iterated until the network converges.

A list of the training algorithms that are available in the Neural Network Toolbox software and that use gradient method is shown in the following table II.

TABLE II. GRADIENT METHOD

\begin{tabular}{|l|l|}
\hline \multicolumn{1}{|c|}{ Function } & \\
\hline trainlm & Llgorithm \\
\hline trainbr & Bayesian Regularization \\
\hline trainbfg & BFGS Quasi-Newton \\
\hline trainrp & Resilient Back propagation \\
\hline trainscg & Scaled Conjugate Gradient \\
\hline traincgb & Conjugate Gradient with Powell/Beale Restarts \\
\hline traincgf & Fletcher-Powell Conjugate Gradient \\
\hline traincgp & Polak-Ribiére Conjugate Gradient \\
\hline trainoss & One Step Secant \\
\hline traingdx & Variable Learning Rate Gradient Descent \\
\hline traingdm & Gradient Descent with Momentum \\
\hline traingd & Gradient Descent \\
\hline
\end{tabular}

5) Click the "Train" button of Fig.6, generate the training model, which performance as shown Fig .7.

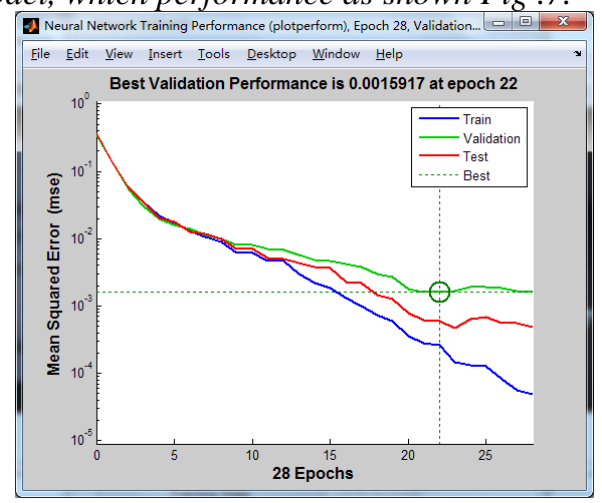

Figure 7. the result of training model performance 


\section{RECOGNITION ALGORITHM AND RESULT}

A. Segment the Character Image of ID Code Accordig to the Steps of TABLE I.

In order to verify algorithm of the training mode, identified ID card should be segment the character image according to the steps of Table I.

Fig .8 shows the characters image result of segmentation of ID card code. Each character image is shown below in the text box.

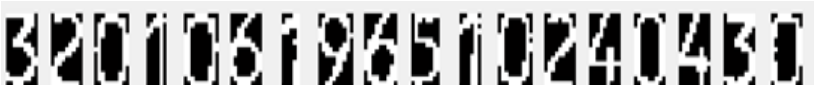

Figure 8. the characters image result

\section{B. Using the Generated the Training Model to Identify Code of the ID Card}

The recognition algorithm is based on the generated the training model to identify code of ID cards. Fig .9 shows the recognition result of segmentation of ID card code. Each recognition result is shown below in the text box.

\begin{tabular}{|l|l|l|l|l|l|l|l|l|l|l|l|l|l|l|l|l|l|}
\hline 3 & 2 & 0 & 1 & 0 & 6 & 1 & 9 & 6 & 5 & 1 & 0 & 2 & 4 & 0 & 4 & 3 & 0 \\
\hline
\end{tabular}

Figure 9. the recognition result

\section{VALIDATION ALGORITHM AND RESULT}

In order to verification convenience, filename of ID card image is named as same as code of ID card. If character of text box in Fig .9 is not same as code of ID card, the character will be display by red color. Fig .10 shows the verification result of ID card code, which has error code marked by red color.

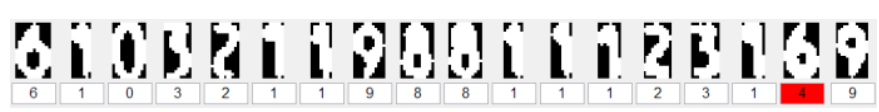

Figure 10. the verification result has error code

In order to verify recognition accuracy rate, Fig .11 shows the verify recognition accuracy rate. ID cards successful rate and codes successful are computed.

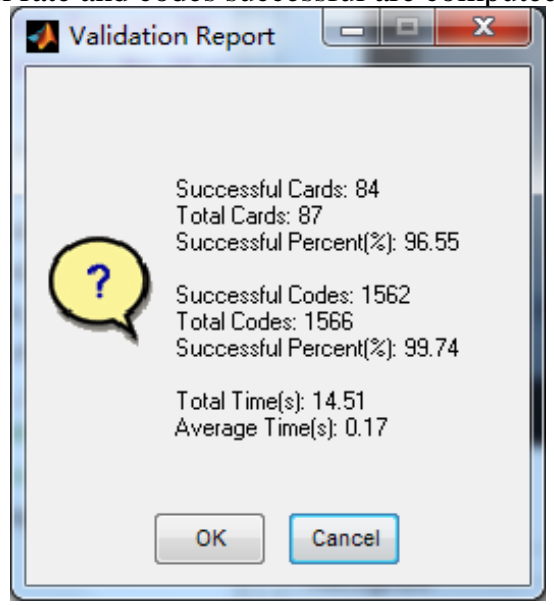

Figure 11. verify recognition accuracy rate

There are total 87 second-generation ID cards which have been verified. ID cards of 84 are correctly identified. The correct recognition rate is 96.55 percent.
There are total 1566 code characters which have been verified. Characters of 1562 are correctly identified. The correct recognition rate is 99.74 percent.

The total processing time is 14.51 seconds. The average processing time for each second-generation ID card image is 0.17 seconds.

\section{CONCLUSION}

The three algorithms of training, recognition and validation have been depicted in the paper. Because the training model is base of ID cards recognition, so the important algorithm is training algorithm which is basis of other algorithms.

Training model is involving in parameters configuration of neural network function. If ID card has been wrongly identified, you need to mark the wrong recognition character, which should be stored in the sample library of character images. The sample data has been added in order to generate the excellent training model which can enhance the recognition accuracy rate of ID cards. This process can continue to cycle until the train recognition rate model substantially the required accuracy.

On the other hand, if the origin ID cards is scanned clearly and distinctly, the recognition accuracy rate can extremely be raised using training model.

The algorithms have been proposed can take advantage of the training model to perfectly realize identification and validation the code of the second-generation ID card; the algorithms have application value in the real work.

\section{REFERENCES}

[1] Gao Ju, Ye Hua, Yang Yan Lan. Android platform identification ID number [J]. of information technology and informationization based on 2013,04:116-119+122

[2] Le Kai, Chen Lian, Cao Jichang. Based on the gray multi value ID card number recognition[J].computer engineering and applications,:

[3] Hong Yamin. The two generation ID card photo identification [J] Journal of Lanzhou industry college, 2013,06:18-22.

[4] Zhao Xingwang, Li Tianyang, Wang Liang, Zhou Jing. The two generation ID card number recognition system of [J]. computer and modernization based on digital equipment, 2014,06:132-136.

[5] Qiu Dao Yin, Liu Qingxia. The recognition algorithm MATLAB. Journal of the identity card numbers of North China Institute of water conservancy and hydroelectric power based on $[\mathrm{J}]$. 2012,02:91-94.

[6] Junhuan Zhou (Wuhan Univ. of Technol., Wuhan, China); Jingling Yuan; Haizheng Tao; Hui Huang; Wu Qiu Source: 2013 8th International Conference on Computer Science \& Education (ICCSE 2013), p 769-72, 2013

[7] Supardi, J. (Inf. Eng. Dept., Sriwijaya Univ., Sriwijaya, Indonesia); Utami, A.S. Source: International Journal of Machine Learning and Computing, v 4, n 1, p 110-13, Feb. 2014

[8] Graditi, G. (ENEA - Res. Center, Italian Nat. agency for new Technol., Portici, Italy); Ferlito, S.; Adinolfi, G.; Tina, G.M.; Ventura, C. Source: 2014 5th International Renewable Energy Congress (IREC), p 6 pp., 2014

[9] Sivanandan, S., Saiyyad, Y.: Automatic vehicle identification using license plate recognition for Indian vehicles. Int. J. Comput. Appl.2012, 23-28 (2012)

[10] Ibrahim, N.K., Kasmuri, E., Norazira, A.: License plate recognition (LPR): a review with experiments for Malaysia case study. Int. J. Soft Comput. Softw. Eng. 3 (3), (2013)

[11] Dandu, B.R., Chopra, A.: Vehicular number plate recognition using edge detection and characteristic analysis of national number plates. Int. J. Comput. Eng. Res. 2 (3), 795-799 (2012) 